\title{
An Examination of Abnormal Grain Growth in Low Strain Nickel-200
}

\author{
O. Underwood ${ }^{1} \cdot$ J. Madison ${ }^{1}$ R. M. Martens ${ }^{2}$ - G. B. Thompson ${ }^{3}$. \\ S. Welsh ${ }^{4} \cdot$ J. Evans ${ }^{4}$
}

Received: 14 April 2016/Revised: 20 May 2016/Accepted: 25 May 2016/Published online: 21 June 2016

(C) The Author(s) 2016. This article is published with open access at Springerlink.com

\begin{abstract}
This study offers experimental observation of the effect of low strain conditions $(\varepsilon<10 \%)$ on abnormal grain growth (AGG) in Nickel-200. At such conditions, stored mechanical energy is low within the microstructure enabling one to observe the impact of increasing mechanical deformation on the early onset of AGG compared to a control, or nondeformed, equivalent sample. The onset of AGG was observed to occur at specific pairings of compressive strain and annealing temperature and an empirical relation describing the influence of thermal exposure and strain content was developed. The evolution of low- $\Sigma$ coincident site lattice (CSL) boundaries and overall grain size distributions are quantified using electron backscatter diffraction preceding, at onset and during ensuing AGG, whereby possible mechanisms for AGG in the low strain regime are offered and discussed.
\end{abstract}

Keywords Nickel · Abnormal grain growth · Low strain · Electron backscattered diffraction

O. Underwood

odunder@sandia.gov

1 Materials Mechanics, Sandia National Laboratories, Albuquerque, NM 87185-0889, USA

2 Central Analytical Facility, The University of Alabama, Tuscaloosa, AL 35487, USA

3 Metallurgical and Materials Engineering, The University of Alabama, Tuscaloosa, AL 35487, USA

4 Materials Science Program, University of Alabama in Huntsville, Huntsville, AL 35899, USA

\section{Introduction}

Mechanical properties are often closely associated with microstructure, wherein grain size, shape, and orientation are often predominant factors in the determination of mechanical strength. At elevated temperatures, grains can acquire sufficient thermal energy to coarsen or grow. This behavior can be further accelerated when the material has or is undergoing some form of mechanical deformation. In these cases, stored energy increases the driving force for microstructural evolution. A phenomenon referred to as abnormal grain growth (AGG) occurs when grain coarsening is not self-similar and a specific subset of grains grow at a rate faster than others. As one might expect, AGG has been shown to have detrimental impacts on mechanical properties [1-5]. For example, Flageolet et al. showed fatigue life in polycrystalline nickel-based superalloy 718 decreased by as much as an order of magnitude in the presence of AGG [2]. Gabb et al. reported a similar reduction in fatigue life for a low solvus high refractory (LSHR) nickel-based superalloy when abnormally large grains were present within the microstructure [5]. While there still exists much debate regarding the specific mechanisms for the onset of AGG, most authors agree that the formation of abnormal grains is commonly related to the selective migration of low-energy or high mobility grain boundaries [6-10].

An outcome of certain grain boundary characterization efforts [3, 11, 12] has been the concept of Grain Boundary Engineering (GBE) to bolster grain stability against AGG or other undesirable networks of grain orientations and interfaces. This concept was proposed by Watanabe in 1984 wherein it was suggested that properties of metallic systems could be enhanced by controlling the distribution of grain boundary types and increasing the frequency of 
specific grain boundaries using thermomechanical processing [13]. This type of processing utilizes varying combinations of work hardening (e.g., cold rolling, tensile straining, compressive straining, etc.) in conjunction with subsequent or intermittent heat treatment to alter microstructure and thus improve material response $[14,15]$. While a multitude of GBE methods exist, one could categorize GBE efforts within one of two main processing regimes, regardless of the thermal cycle used. For this discussion, the authors consider this binary categorization as low strain (where induced $\varepsilon<10 \%$ per cycle) and high strain (where induced $\varepsilon>10 \%$ per cycle) [15]. In this work, the focus is within the low strain regime.

The vast majority of research in GBE and AGG has involved materials subjected to higher strains [8, 11, 16, 17], where stored mechanical energy is large and facilitates a greater driving force for microstructural change in grain structure. In contrast, at low strains, one can capture how intrinsic grain boundary evolution is influenced by minor deformations and its coupled effect on AGG. To study this behavior, commercially pure $\mathrm{Ni}-200$ was selected as the material system of investigation as Nickel has been shown to be susceptible to AGG [18-20] and possesses a wellcharacterized grain structure for comparison. Furthermore, nickel is a tremendously ubiquitous material system as it is a primary constituent found in many of the most advanced alloy systems developed to date, where the applications of such alloys include turbine blades for aircraft engines, land-based power turbines, as well as heat transfer and cooling systems in nuclear and chemical processing plants $[1,21,22]$.

\section{Experimental}

A series of Ni-200 bars having dimensions of $152 \times$ $6.35 \times 6.35 \mathrm{~mm}$ were cold rolled under multiple rolling passes to achieve desired reductions of 3, 6, and 9\% using a Stanat Model: TA 215, rolling mill. After rolling to the desired strain, plates were subjected to a thermal anneal for $30 \mathrm{~min}$ at a specific temperature within the range of 200-800 ${ }^{\circ} \mathrm{C}$ under a flowing argon-rich atmosphere to prevent oxidation. In general, the furnace dwell temperature was achieved prior to the insertion of the samples. The only modification to this procedure occurred during cyclic annealing of the $9 \%$ strain cases. Due to a change in experimental apparatus, these samples were present within the furnace during the heating ramp and then held at the desired dwell temperature for $30 \mathrm{~min}$ within a flowing hydrogen-rich atmosphere to again reduce opportunity for oxidation. It was found that noticeable changes in grain behavior occurred at temperatures greater than $700{ }^{\circ} \mathrm{C}$. Consequently, this paper will only present those findings wherein successive increments of $20{ }^{\circ} \mathrm{C}$ were used in the evaluation of cyclic annealing response. The reader is directed to Ref. [19] for a comprehensive report of the effect of strained $\mathrm{Ni}-200$ subjected to lower temperature anneals.

Once rolled and annealed, plates were metallographically polished to a $0.05 \mu \mathrm{m}$ colloidal silica finish where samples surfaces were prepared parallel to the rolling direction. After rolling, grain size remained relatively unchanged across 0,3 , and $6 \%$ strain but was observed to increase by a factor of nearly 2 for the cases of $9 \%$ strain. Once polished, electron backscattered diffraction (EBSD) was employed to examine the same regions of interest (ROI) and track specific grain boundary and texture evolutions at each thermal increment. ROIs were designated by fiducial markers allowing repeated imaging of identical areas. EBSD data collection occurred at three different facilities; therefore, the operating conditions varied slightly depending on the scanning electron microscope (SEM) used. Typically, the SEM beam current was 4 nA. Camera binning size of either $4 \times 4(160 \times 120)$ or $6 \times 6$ $(106 \times 80)$ was used for nearly all cases. TSL orientation imaging microscopy analysis by EDAX, Inc. was used to analyze all EBSD data acquired. For orientation mapping, a step size of 0.5 or $1 \mu \mathrm{m}$ was used over multiple crosssectional areas of $500 \times 500 \mu \mathrm{m}$ or larger. The Brandon criterion (where $\theta=15^{\circ} \Sigma 1^{-1 / 2}$ ) was used to determine the maximum permissible deviation from coincidence to characterize the coincident site lattice (CSL) boundaries [23]. Within each initial ROI, at least 200 grains were identified and used to quantify the grain boundary character. Neighbor Confidence Index (CI) correlation followed by grain CI standardization cleanup processes were used for all four samples. Average confidence index of $>0.1$ was used. Figure 1 shows a schematic of the cyclic annealing and EBSD schedule used in this study.

\section{Results and Discussion}

As a control, grain boundary metrics for the unstrained "asreceived" Nickel-200 were annealed and metallographically prepared parallel to the rolling direction as shown in Fig. 2(a)-(d). Mean grain size diameter, inclusive of twins, was determined to be $7 \mu \mathrm{m}$ with a near Gaussian distribution, while the grain size occupying the greatest area fraction was approximately $15 \mu \mathrm{m}$, see Fig. 2(c). In Fig. 2(d), CSL twin boundary populations are shown wherein $\Sigma 3$ boundaries are highlighted in red, $\Sigma 9$ in blue, and $\Sigma 27$ in green. Low CSL boundaries not belonging to these specific special boundaries are highlighted in black and are denoted in the legend. In the unstrained state, special grain boundaries $(3<\Sigma<29)$ composed $55.4 \%$ of 




Fig. 1 Cyclic annealing schedule used where $T_{\text {start } 1}=700{ }^{\circ} \mathrm{C}$ and $T_{\text {start } 6}=800{ }^{\circ} \mathrm{C}$. Each cycle was concluded with EBSD scan on preselected regions of interest for tracking grain and grain boundary evolution

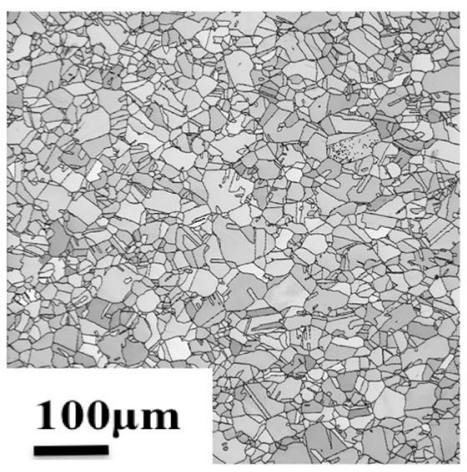

(a)

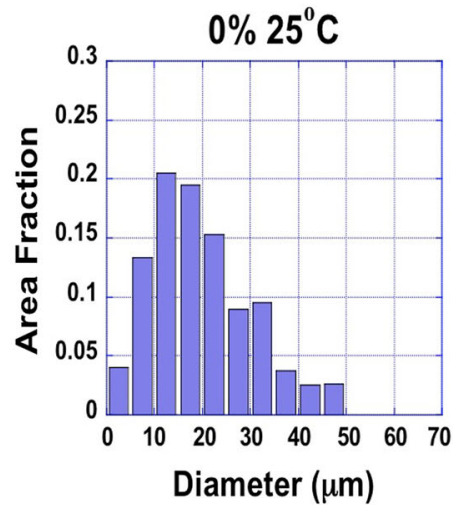

(c)



(b)

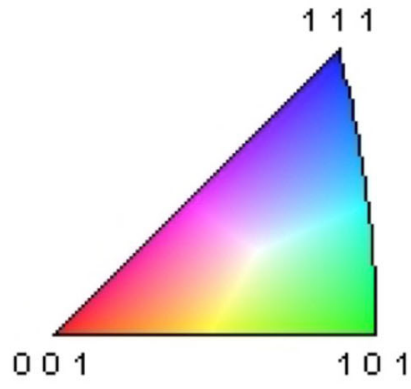

Sigma $(\Sigma)$



(d)



Fig. 2 Unstrained, room temperature baseline grain boundary metrics for commercially pure Ni-200 sectioned parallel to rolling direction. (a) EBSD grain contrast map, (b) grain orientation map with accompanying Inverse pole figure (IPF), (c) grain area fraction distribution, and (d) coincident site lattice (CSL) map with $\Sigma 3, \Sigma 9$ and $\Sigma 27$ highlighted by red, blue, and green, respectively 
all grain boundaries imaged, with the remaining $44.6 \%$ being random. Of the $55.4 \%$ of special grain boundaries present, $51.5 \%$ belong to $\Sigma 3, \Sigma 9$, or $\Sigma 27$ designations with the balance being $\Sigma 5, \Sigma 7, \Sigma 11, \Sigma 13 \mathrm{a}, \Sigma 13 \mathrm{~b}, \Sigma 15, \Sigma 17 \mathrm{a}$, $\Sigma 17 \mathrm{~b}, \Sigma 19 \mathrm{a}, \Sigma 19 \mathrm{~b}, \Sigma 21 \mathrm{a}, \Sigma 21 \mathrm{~b}, \Sigma 23, \Sigma 25 \mathrm{a}, \Sigma 25 \mathrm{~b}, \Sigma 27 \mathrm{a}$, $\Sigma 27$ b, $\Sigma 29$ a, or $\Sigma 29$ b. The $9 \%$ strain case showed equivalent distributions with the only difference being a mean grain size diameter of $14 \mu \mathrm{m}$ as opposed to $7 \mu \mathrm{m}$, and the greatest area fraction contributing grain size was approximately $30 \mu \mathrm{m}$.

A series of EBSD maps for a given ROI at an initial induced strain of $0,3,6$, and $9 \%$ are shown in Fig. 3 as a function of annealing dwell temperature. Each row depicts the same ROI at a specific induced strain level, while each column displays the given ROI following a specific annealing treatment as denoted by the temperature heading for each column. As the initial strain increased, the initiation of AGG was observed to occur at successively lower temperatures. As shown in Fig. 3, AGG was observed to initiate at $780{ }^{\circ} \mathrm{C}$ for $0 \%$ strain; $760{ }^{\circ} \mathrm{C}$ for $3 \%$ strain; $740{ }^{\circ} \mathrm{C}$ for $6 \%$ strain; and $720{ }^{\circ} \mathrm{C}$ for $9 \%$ strain. Dashedline boxes in Fig. 3 are used to denote the regions undergoing AGG.

Quantitatively, this onset of AGG can be discerned by comparing the cumulative grain size area fractions for each case as shown in Fig. 4. With exception to the 9\% induced strain case, all baseline cumulative area fractions displayed a narrow grouping of increasing area fraction in which



(a)

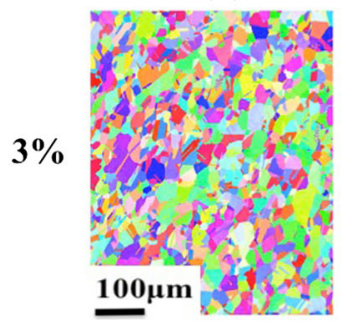

(g)

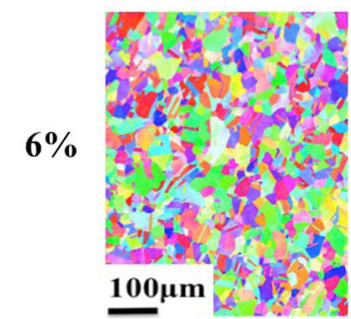

(k)



(o)

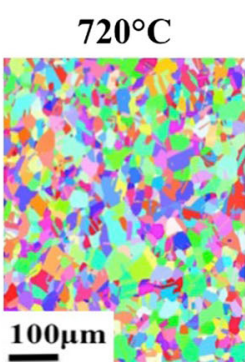

(b)

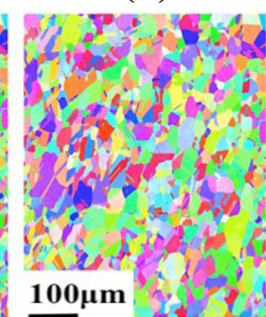

(h)

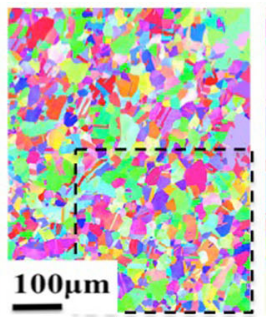

(I)



(p)



(c)

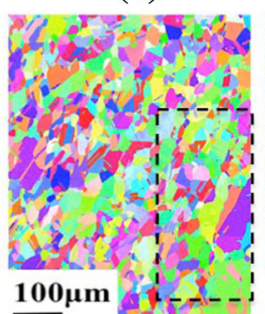

(i)



(m)

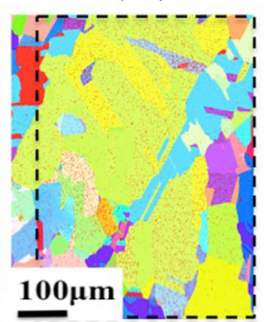

(q)

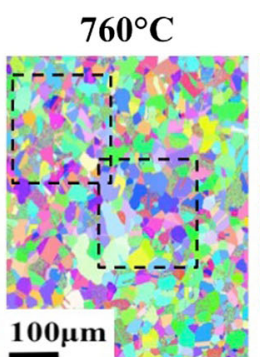

(d)

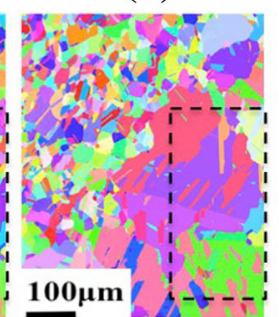

(j)

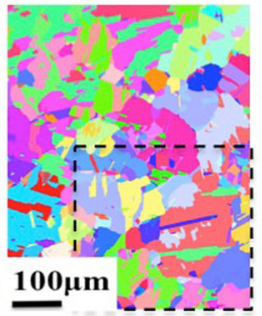

(n)

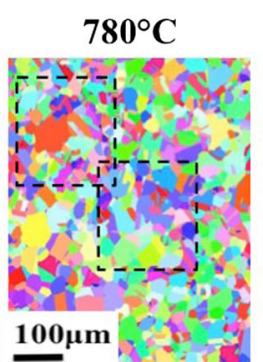

(e)

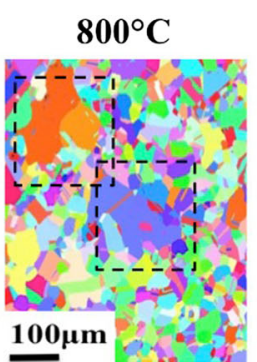

(f)

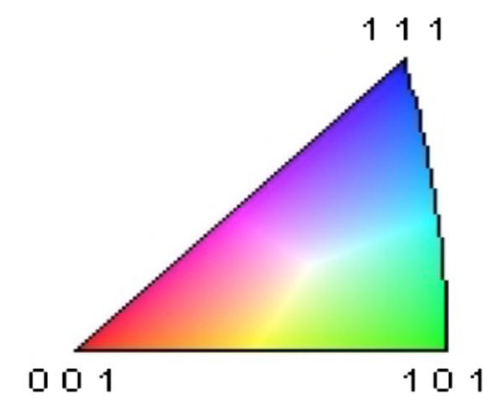

Fig. 3 Orientation imaging microscopy progression maps for $0,3,6$, and $9 \%$ induced strain following annealing treatments ranging from 700 to $800{ }^{\circ} \mathrm{C}$ 


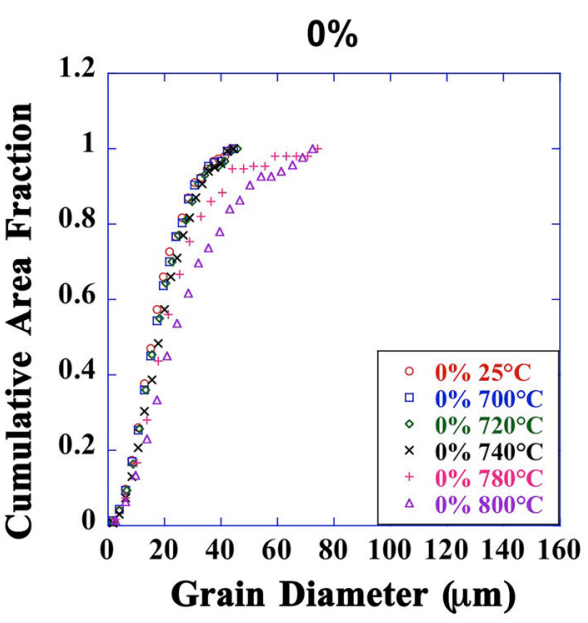

(a)

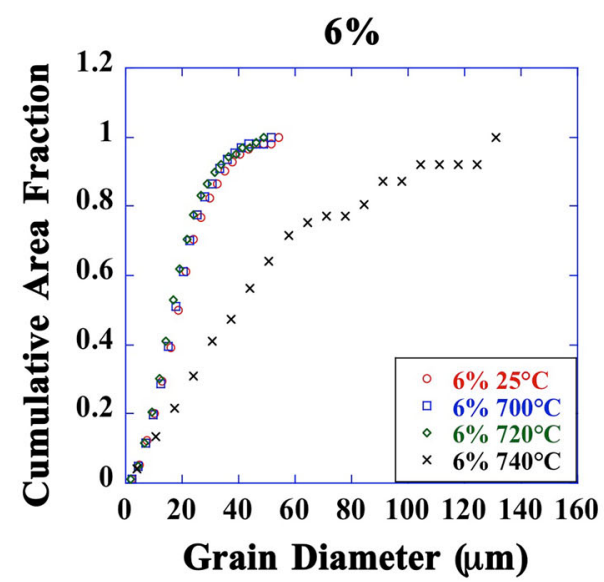

(c)



(b)

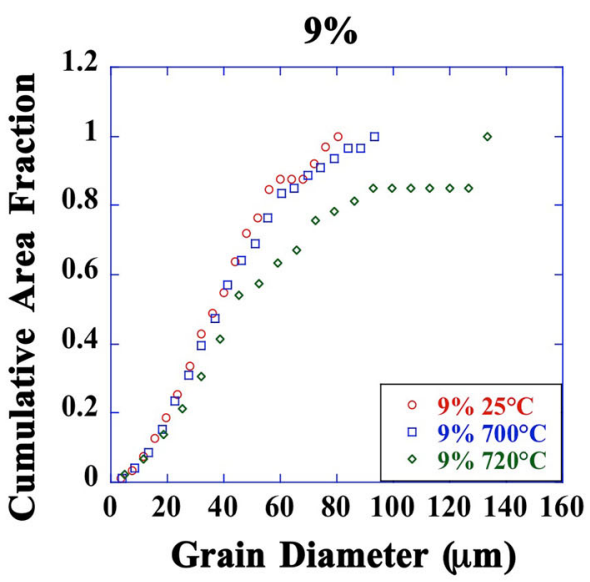

(d)

Fig. 4 Cumulative area fraction grain diameters for (a) $0 \%$, (b) 3\%, (c) 6\%, and (d) $9 \%$ ROIs as a function of temperature

nearly $100 \%$ of all grain sizes are accounted for at or near a threshold grain diameter of $50 \mu \mathrm{m}$ prior to AGG. This is also in agreement with the grain size distribution of the asreceived Ni-200, as seen in Fig. 2(c) prior to annealing, wherein the upper tail of the distribution terminates in the vicinity of $50 \mu \mathrm{m}$. For the $9 \%$ case, the upper threshold value was in the vicinity of $\sim 80-90 \mu \mathrm{m}$. This collective grouping of grain sizes was consistent across all strains and temperatures up to $720{ }^{\circ} \mathrm{C}$. However, for the $0,3,6$, and 9\% strain cases, this grouping in the distributions began to deviate substantially at $780,760,740$, and $720{ }^{\circ} \mathrm{C}$ respectively, see Fig. 4. These departures are symptomatic of the AGG that was observed in Fig. 3. Correspondingly, as the initial induced strain increased, the deviation from the baseline cumulative area fraction diverges at lower cumulative area fractions as might be expected.

To determine whether AGG was linked simply to an onset temperature or to cyclic annealing, a series of isothermal anneals of equivalently strained material at the observed AGG-onset temperatures were pursued as a comparison. It should be stated that these anneals were carried out in a similar manner to the $9 \%$ strain case in which the samples were present for the heating ramp as well as the isothermal dwell. As shown in Fig. 5, over multiple repeated tests, EBSD results revealed no AGG in any case of the isothermally aged and strained $\mathrm{Ni}-200$. The only differences observed between isothermal cases was in the post anneal average grain size, which appeared to correspond to the degree of initial compressive strain imparted into the samples with higher compressive strains resulting in lower post isothermal anneal grain sizes. A summary of mean grain size across all annealing tests is presented in Table 1. From the data, it can be seen there is almost no significant change in average grain size for cyclic annealing cases in which AGG was observed; however, for the isothermal anneals, the mean grain size increased by a factor of $2 \mathrm{X}-3 \mathrm{X}$ with no abnormally large grains being present.

As a visual aid, the plots of AGG and its onset observed are shown in Fig. 6 as a function of annealing temperature 


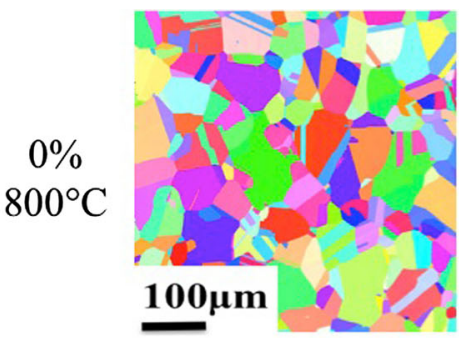

(a)

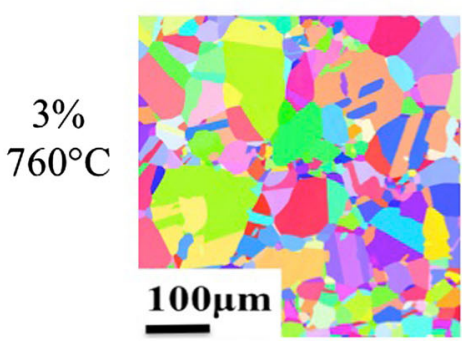

(e)

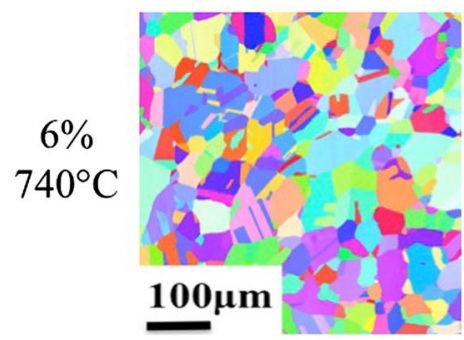

(i)



(b)

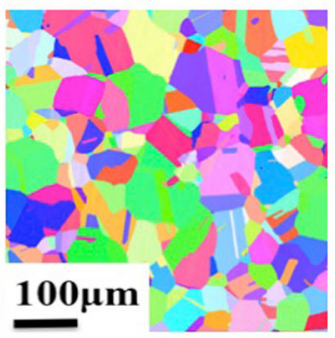

(f)

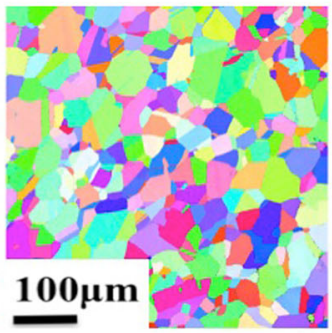

(j)

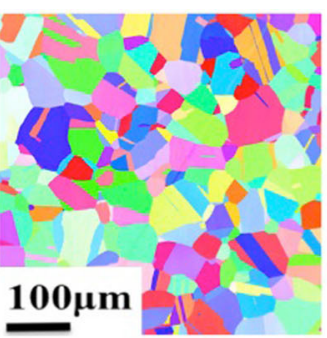

(c)



(g)

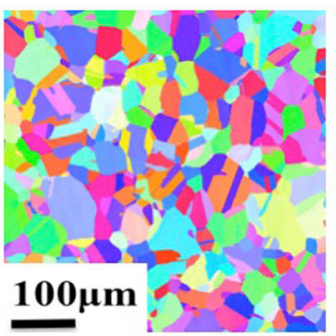

(k)

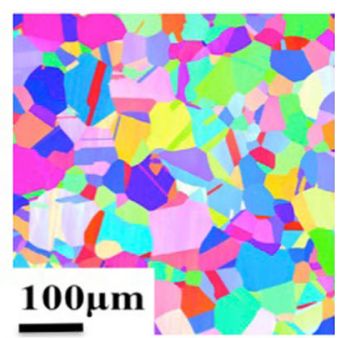

(d)

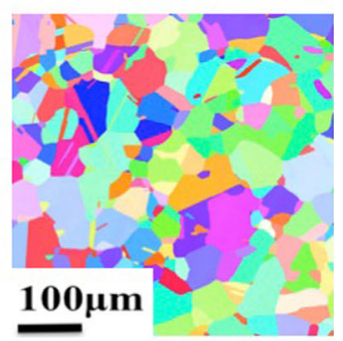

(h)

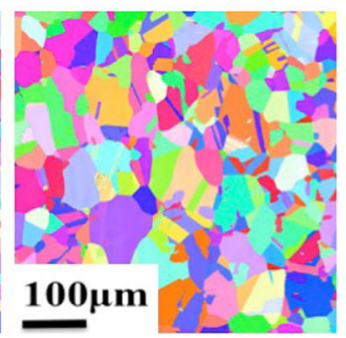

(l)

Fig. 5 EBSD maps of isothermally annealed Ni-200 at (a-d) 0\%, (e-h) 3\% and (i-l) 6\% compressive strain at temperatures for which AGG was observed following cyclic annealing. Each letter correspond to a unique test case at a specific strain level under a single isothermal anneal

Table 1 Measured average grain size

\begin{tabular}{lllll}
\hline $\begin{array}{l}\text { Induced compressive } \\
\text { strain }(\%)\end{array}$ & $\begin{array}{l}\text { Initial avg. grain } \\
\text { size }(\mu \mathrm{m})\end{array}$ & $\begin{array}{l}\text { Thermal anneal } \\
\left({ }^{\circ} \mathrm{C}\right)\end{array}$ & $\begin{array}{l}\text { Isothermal anneal avg. grain size }(\mu \mathrm{m}) \\
{[\text { measured })}\end{array}$ & $\begin{array}{l}\text { Cyclic anneal avg. grain size }(\mu \mathrm{m}) \\
{[\mathrm{measured}]}\end{array}$ \\
\hline 0 & 7.1 & 800 & 20.4 & 8.6 \\
3 & 7.4 & 760 & 16.3 & 7.6 \\
6 & 7.6 & 740 & 14.9 & 7.5 \\
9 & 13.8 & 720 & $\ldots$ & 12.3 \\
\hline
\end{tabular}

and initial induced strain content. This parameter map forms the basis for the empirical relation provided in Eqs. 1 and 2 .

In an effort to incorporate the effect of annealing cycles, the total number of cycles experienced $(n)$ can be written as a function of the induced compressive strain:

$n=5-\left(\varepsilon_{\mathrm{c}} / 3\right)$,

where $n$ is the number of annealing cycles and $\varepsilon_{\mathrm{c}}$ is the initial induced compressive strain. Rewriting for the compressive strain in terms of the number of cycles and substituting into the observed behavior, the following empirical relation was derived:

$T_{\mathrm{AGG}} \geq 20\left(5-\frac{\varepsilon_{\mathrm{c}}}{3}\right)+680$,

where $T_{\mathrm{AGG}}$ is the minimum annealing temperature at which observations of AGG occurred. In this study, while annealing cycles were discontinued once AGG was confirmed, the available data suggest AGG would continue with successive anneals within this temperature range and potentially beyond [19]. This formulation is similar to that 




Fig. 6 AGG onset as a function of strain and annealing temperature

which was proposed by Guyot et al. to derive an empirical relation for the fraction of special grain boundaries produced by cyclic annealing based upon strain, temperature, and number of cycles [15].

Additionally, a comparison of area fractions as a function of grain size between initial and AGG microstructures are shown in Fig. 7(a)-(h). To aid in this comparison, each histogram is plotted on near equivalent axes. As evidenced by the histograms, across all cases of AGG, see Fig. 7(b), (d), (f), and (h), a secondary peak emerges at very large grain sizes, while the overall distribution begins to encompass slightly larger grain sizes yet maintains a relatively unchanged mean value. This fundamental change in the grain size distributions from a unimodal, near Gaussian distribution, to a log-normal distribution with the addition of a pronounced tail provides another quantitative indication of the presence of AGG which may be a more commonly recognized depiction to some.

With regard to grain boundary character, to provide a visual representation of the CSL populations in the vicinity of AGG, the fraction of CSL and twin boundaries $(\Sigma=3)$ were determined by evaluation of the misorientation of adjacent grains. These CSL maps are shown in Fig. 8 for 0 , 3,6 , and $9 \%$ strained $\mathrm{Ni}-200$, with $\Sigma 3, \Sigma 9$, and $\Sigma 27$ boundaries being highlighted in red, blue, and green, respectively. In an effort to help orient the reader, EBSD maps have been included for the cycle immediately prior to the observance of AGG and the matrix of cases being reported are shown in the same manner as done previously in Fig. 3. As can be observed, the overwhelming majority of CSL boundaries across all annealing temperatures and induced strain levels are $\Sigma 3$. While minor fluctuations exist, this observation is consistent even in the regions of AGG as indicated by the dashed boxes.



(a)

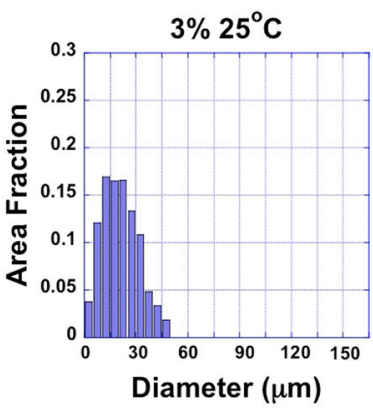

(c)

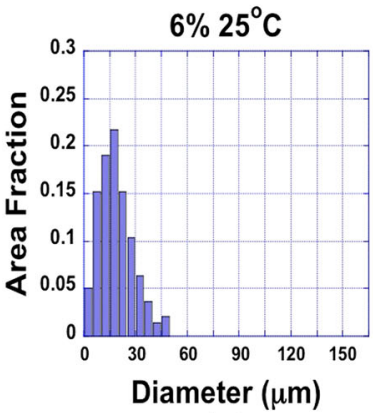

(e)

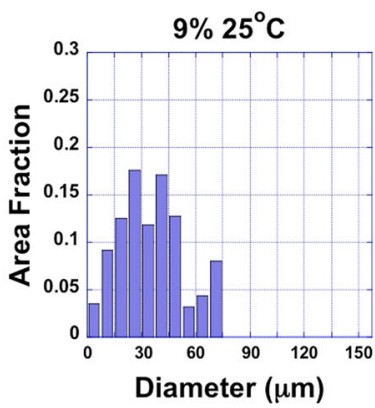

(g)

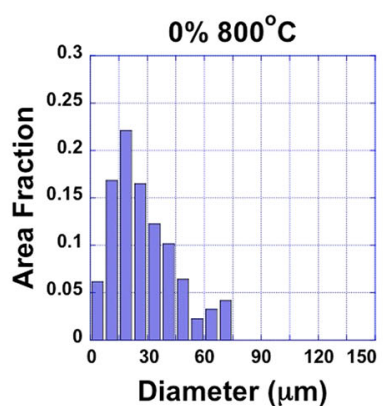

(b)



(d)

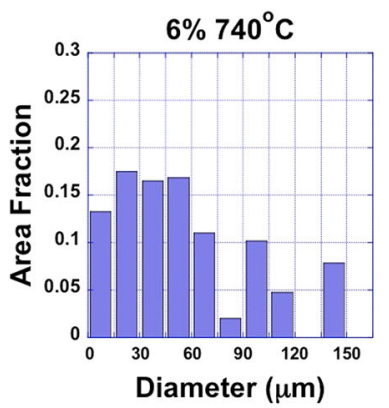

(f)

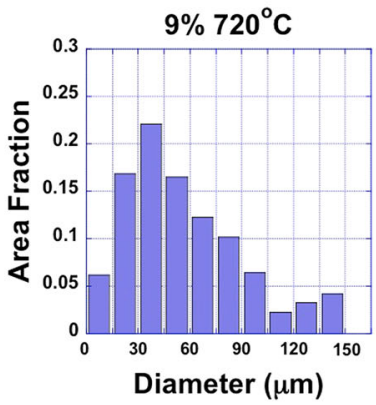

(h)
Fig. 7 Comparison of grain size-based contributions to total area fraction between room temperature and AGG containing microstructures $(\mathbf{a}, \mathbf{b}) \varepsilon_{\mathrm{c}}=0 \%,(\mathbf{c}, \mathbf{d}) \varepsilon_{\mathrm{c}}=3 \%,(\mathbf{e}, \mathbf{f}) \varepsilon_{\mathrm{c}}=6 \%,(\mathbf{g}, \mathbf{h}) \varepsilon_{\mathrm{c}}=9 \%$

However, to quantitatively assess the relative change of special grain boundaries in the vicinity of AGG, boundary fractions of the primary CSL boundaries have been evaluated as a function of annealing cycle, and the plots of the boundary fractions are shown in Fig. 9. The error bars 


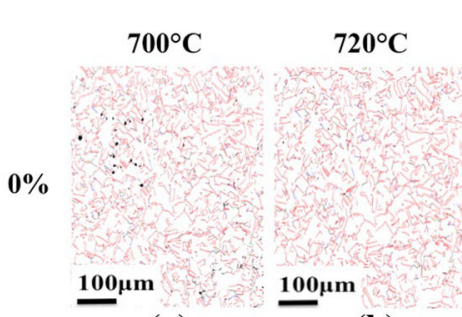

(a)



(g)

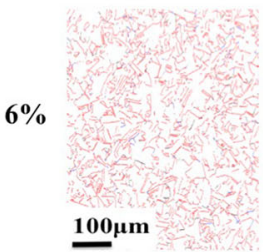

(k)

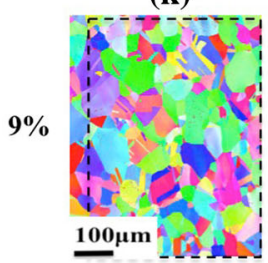

(o) (b)

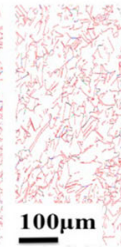

(h)

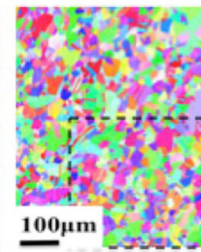

(I)



(p)

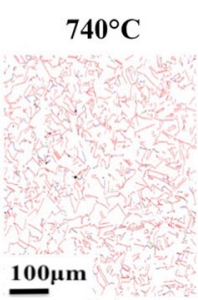

(c)

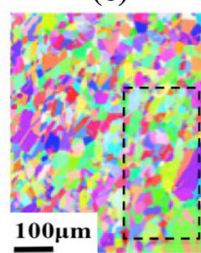

(i)

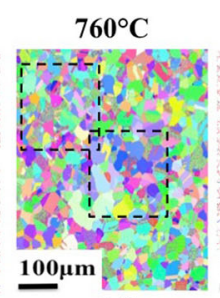

(d)



(j)



(e)

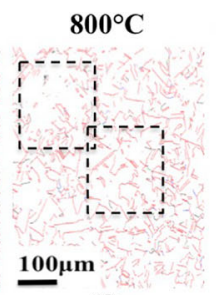

(f)

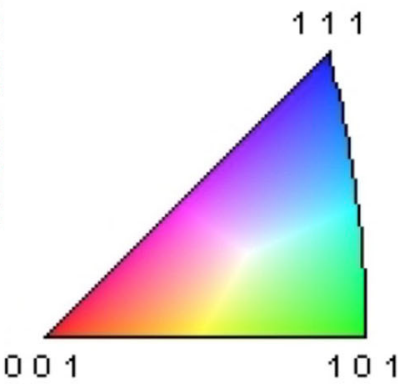

\section{$\underline{\operatorname{Sigma}(\Sigma)}$}

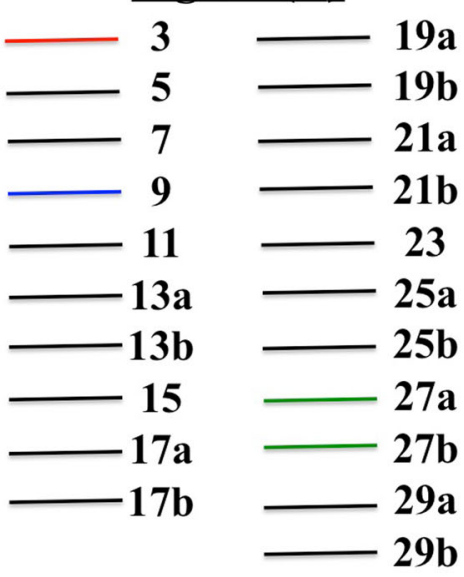

Fig. 8 CSL boundary maps determined by evaluation of misorientation between adjacent grains. $\Sigma 3, \Sigma 9$, and $\Sigma 27$ boundaries are highlighted in red, blue, and green, respectively. Across all compressive strains and all annealing cycles, $\Sigma 3$ boundaries exist in the highest populations even in the areas of AGG

maxima of $\Sigma 3, \Sigma 9$, and $\Sigma 27$ for the 3, 6, and $9 \%$ cases. Alternatively, for the $0 \%$ case, the maximum fraction of $\Sigma 1$ and the minimum fraction of $\Sigma 3$ were observed at $800{ }^{\circ} \mathrm{C}$. Lastly, since data measurements were available for analysis of multiple cycles following the onset of AGG for 6 and $9 \%$, it was also observed in these cases that as AGG continued, $\Sigma 3$ boundary fractions exhibited a local decrease after the onset of AGG. For $0 \%$ compressive strain cases, no significant variation was observed for any member of the $\Sigma\left(3^{n}\right)$ class of grain boundaries.

\section{Discussion}

In this study, increased initial compressive strain was observed to decrease the onset temperature for AGG following cyclic annealing, even at low strain values. Knowledge of these onset conditions can allow others to perform GBE in Ni-200 at low strain with the goal of either avoiding the initiation of AGG or targeting its occurrence if the observed AGG-onset temperatures and/or the local 


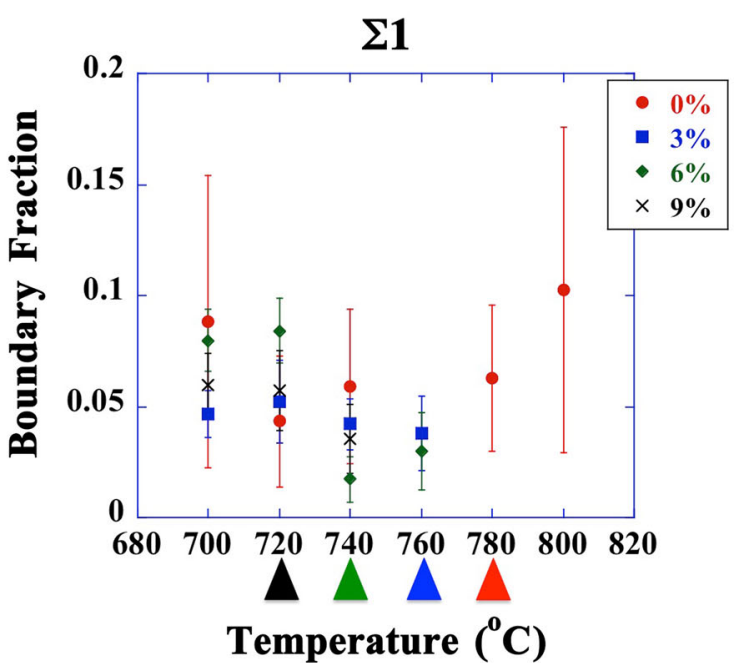

(a)



(c)

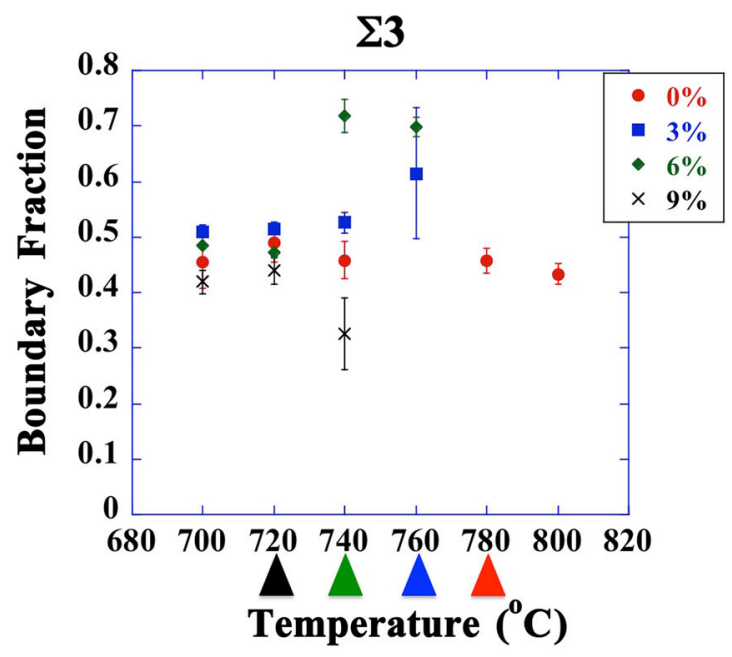

(b)

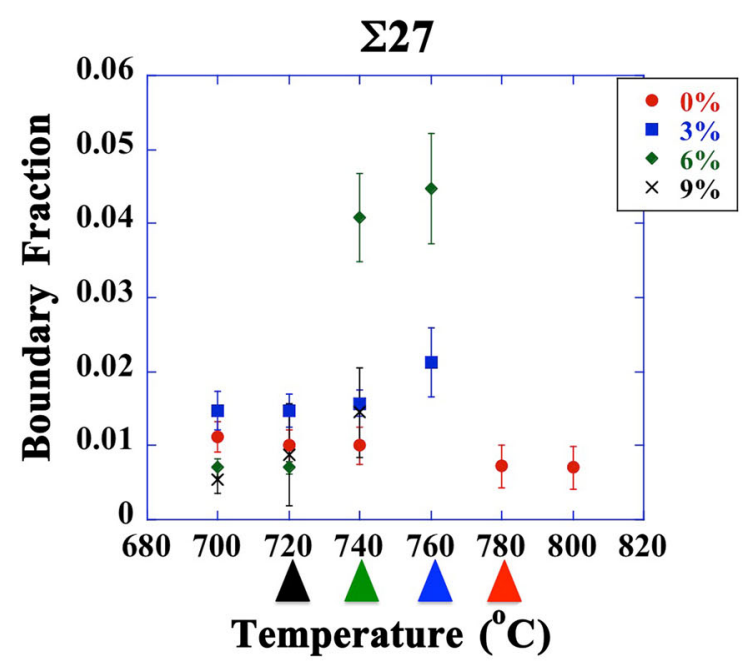

(d)

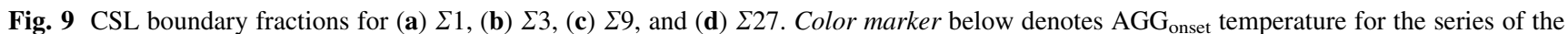
equivalent color

so desired. As mentioned previously, $\Sigma 3, \Sigma 9$, and $\Sigma 27$ demonstrated local maxima at or immediately following AGG, followed by a subsequent decrease in their relative quantity as AGG ensued for 3,6, and $9 \%$ cases. Similar results, showing a loss of $\Sigma 3$ grain boundary length after the onset of AGG, were observed in a companion study performed by Brons et al. [26] who hypothesized the high mobility of the $\Sigma 3$ boundaries allowed for AGG. It was also shown by Brons et al. that at the onset of AGG, there was a distinct local increase in the presence of $\Sigma 3$ and $\Sigma 9$ boundaries [26]. Although this study was performed on undeformed nanocrystalline metallic systems of nickel, the findings of this study, while observed at the mesoscale, are complimentary in the aforementioned observations. Conversely, Randle and Horton [18] observed the microstructures of high purity undeformed nickel, to undergo AGG after annealing for several hours under vacuum and then returning to typical grain growth after additional thermal exposure in the range of $900{ }^{\circ} \mathrm{C}$. Here, this study revealed AGG can occur at $0 \%$ strain following cyclic annealing for very short durations and did not require being preceded by hours of thermal exposure.

When comparing the outcomes of the current study with the results of comparable thermomechanical studies performed at higher strains among materials of equivalent characteristic length scales, similarities are readily observed. One such similarity is strain increase precipitates a decrease in the onset temperature for AGG $[8,16]$ as shown. Furthermore, higher strain studies have also reported that cyclic annealing under varying temperatures 
and times can encourage a transition from typical to AGG in carburizing and noncarburizing environments [8]. Lastly, high strain studies on polycrystalline alloys have also shown slight decreases in the fraction of special grain boundaries while in the presence of AGG [11]. This phenomenon was also observed in this study and is reported above.

The contrast of average grain size measurements achieved via cyclic annealing versus isothermal annealing suggests variations in grain growth behavior are introduced by the cyclic annealing process. Sahay et al. suggest these departures from classical "quasi-isothermal" grain growth kinetics observed during cyclic annealing are largely a result of nonisothermal excitation resulting in enhanced grain boundary mobility which in turn provides a reduction in activation energy for grain boundary motion [17]. As the overall average grain size was not shown to deviate significantly in any cyclic annealing case, even in the presence of AGG, the results of this study suggest that many of the factors posited by Sahay et al. were preferentially enacted in grain boundaries experiencing AGG. These observations of selective grain growth are in direct contrast to the cases of singular isothermal annealing shown here as a comparison, see Fig. 5. In isothermal anneals, mean grain size was shown to increase by $2-3 \mathrm{X}$ their baseline value homogeneously after one isothermal anneal (see Fig. 6; Table 1). However, the cyclic annealing used in this study appears to have selectively provided the thermal excitation necessary for grain arrangements favorable for grain growth while not providing enough energy for other grain boundary networks. This suggests the repeated thermal reversals are sufficient to provide selective excitation. This further corresponds to the findings of Sahay et al. whom prescribed high ramp rates and temperature reversals were significant contributors to increased grain mobilities beyond those observed in isothermal scenarios [17].

\section{Conclusions}

At very low strain conditions, Ni-200 is able to undergo AGG due to the accumulated energy from deformation and thermal excitation. This study records the specific pairings of annealing temperature and imposed strain for the onset and continuation of AGG in the low strain regime. The following are a series of observations and determinations derived from this study:

- As imposed strain was increased, temperature for the onset of AGG decreased to successively lower temperatures during cyclic heat treatment.
- Cumulative area fractions and grain size distributions confirmed a clear correlation between $\mathrm{AGG}_{\text {onset }}$, annealing temperature, and compressive strain.

- An empirical predictor for the onset of AGG was derived as a function of annealing temperature, quantity of thermal cycles imposed, and the initially induced compressive strain. This empirical model takes the following form: $T_{\mathrm{AGG}} \geq 20\left(5-\frac{\varepsilon_{\mathrm{c}}}{3}\right)+680$, where $T_{\mathrm{AGG}}$ is the AGG observation temperature, $\varepsilon_{\mathrm{c}}$ is the initial induced compressive strain, and $\varepsilon_{\mathrm{c}}$ is related to the number of thermal cycles $(n)$ by the following relation: $n=5-\left(\varepsilon_{\mathrm{C}} / 3\right)$.

- While AGG was observed to occur throughout the low strain regime, $(0<\varepsilon<10 \%)$, anticipated increases of $\Sigma 3, \Sigma 9$, and $\Sigma 27$ were only observed as local maxima and occurred at or immediately after the AGG-onset temperature.

- The onset of AGG in the current study is complimentary to other high strain thermomechanical treatments in terms of the qualitative correspondence of AGG with temperature, strain, and thermal cycling. However, low strain Nickel-200 appears to have a higher susceptibility to AGG at shorter thermal dwell times when compared to scenarios of high strain.

Acknowledgments Sandia National Laboratories is a multiprogram laboratory managed and operated by Sandia Corporation, a wholly owned subsidiary of Lockheed Martin Corporation, for the U.S. Department of Energy's National Nuclear Security Administration under Contract DE-AC04-94AL85000. A subset of experiments for this work was supported by the National Science Foundation under Grant No. DMR-1151109. Compressive rolling and initial metallographic preparation of samples were conducted at the Redstone Arsenal in Huntsville, AL. A significant amount of electron backscatter diffraction and annealing studies were conducted at the Center for Nanophase Materials Sciences, a DOE Office of Science, User Facility in Oak Ridge, TN. All other works were performed at Sandia National Laboratories. As such, the authors would like to thank A. Kilgo and D. Renner for sample preparation, C. Walker for annealing studies, as well as B. McKenzie, J. Michael, R. McCabe, and D. Leonard for EBSD experiments.

Open Access This article is distributed under the terms of the Creative Commons Attribution 4.0 International License (http://crea tivecommons.org/licenses/by/4.0/), which permits unrestricted use, distribution, and reproduction in any medium, provided you give appropriate credit to the original author(s) and the source, provide a link to the Creative Commons license, and indicate if changes were made.

\section{References}

1. B.M. Guyot, N.L. Richards, A study on the effect of cold rolling and annealing on special grain boundary fractions in commercialpurity nickel. Mater. Sci. Eng. A 395(1), 87-97 (2005)

2. B. Flageolet, O. Yousfi, Y. Dahan, P. Villechaise, J. Cormier, Characterization of microstructures containing abnormal grain 
growth zones in alloy, in 7th International Symposium on Superalloy 718 (2012), pp. 594-606

3. V. Randle, M. Coleman, Grain growth control in grain boundary engineered microstructures. Mater. Sci. Forum 715-716, 103-108 (2012)

4. R.F. Decker, A.I. Rush, A.G. Dano, J.W. Freeman, Abnormal Grain Growth in Nickel-Base Heat-Resistant Alloys (University of Michigan, Ann Arbor, 1957), pp. 1-72

5. T.P. Gabb, P.T. Kantzos, J. Gayda, C.K. Sudbrack, B. Palsa, Fatigue resistance of the grain size transition zone in a dual microstructure superalloy disk. Int. J. Fatigue 33(3), 414-426 (2011)

6. E.A. Holm, M.A. Miodownik, A.D. Rollett, On abnormal subgrain growth and the origin of recrystallization nuclei. Acta Mater. 51, 2701-2716 (2003)

7. S. Fang, D. Yunpeng, S. Wang, The abnormal grain growth of P/M nickel-base superalloy: strain storage and CSL boundaries. Adv. Mater. Res. 1064, 49-54 (2015)

8. S.B. Lee, N.M. Hwang, D.Y. Yoon, M.F. Henry, Grain boundary faceting and abnormal grain growth in nickel. Metall. Mater. Trans. A 31A, 985-993 (2000)

9. R. Watson, M. Preuss, J.Q. Da Fonseca, T. Witulski, G. Terlinde, M. Buscher, Characterization of abnormal grain coarsening in alloy 718, in MATEC Web of Conferences, vol. 14 (2014), pp. $1-5$

10. C.-S. Park, H.-K. Park, H.-S. Shim, T.-W. Na, C.-H. Han, N.-M. Hwang, New understanding of the role of coincidence site lattice boundaries in abnormal grain growth of aluminum alloy. Philos. Mag. Lett. 95(4), 220-228 (2015)

11. A.J. Schwartz, W.E. King, M. Kumar, Influence of processing method on the network of grain boundaries. Scr. Mater. 54, 963-968 (2006)

12. L. Tan, K. Sridharan, T.R. Allen, Effect of thermomechanical processing on grain boundary character distribution of a Ni-based superalloy. J. Nucl. Mater. 371(1), 171-175 (2007)

13. T. Watanabe, An approach to grain boundary design for strong and ductile polycrystals. Res. Mech. 11(1), 47-84 (1984)
14. S.-L. Lee, N.L. Richards, The effect of single-step low strain and annealing of nickel on grain boundary character. Mater. Sci. Eng. A 390(1-2), 81-87 (2005)

15. B.M. Guyot, N.L. Richards, A preliminary model to predict the special grain boundary fraction in commercial-purity nickel. J. Mater. Process. Technol. 189(1-3), 162-168 (2007)

16. J. Dennis, P.S. Bate, F.J. Humphreys, Abnormal grain growth in metals. Mater. Sci. Forum 558-889, 717-722 (2007)

17. S.S. Sahay, C.P. Malhotra, A.M. Kolkhede, Accelerated grain growth behavior during cyclic annealing. Acta Mater. 51, 339-346 (2003)

18. V. Randle, D. Horton, Grain growth phenomena in nickel. Scr. Metall. Mater. 31(7), 891-895 (1994)

19. O.D. Underwood, An investigation of grain boundary character evolution in nickel 200. PhD thesis, University of Alabama in Hunstville, Alabama. Thesis completed (2015)

20. S.-H. Jung, D.Y. Yoon, S.L. Kang, Mechanism of abnormal grain growth in ultrafine-grained nickel. Acta Mater. 61(15), 5685-5693 (2013)

21. Y. Gao, M. Kumar, R.K. Nalla, R.O. Ritchie, High-cycle fatigue of nickel-based superalloy ME3 at ambient and elevated temperatures: role of grain boundary engineering. Metall. Mater. Trans. A 36(12), 3325-3333 (2005)

22. T.M. Pollock, S. Tin, Nickel-based superalloys for advanced turbine engines: chemistry, microstructure, and properties. J. Propuls. Power 22(2), 361-374 (2006)

23. D.G. Brandon, The structure of high-angle grain boundaries. Acta Metall. 14(11), 1479-1484 (1966)

24. V. Randle, Mechanism of twinning-induced grain boundary engineering in low stacking-fault energy materials. Acta Mater. 47(15), 4187-4196 (1999)

25. L.H. Chan, H. Weiland, S. Cheong, G.S. Rohrer, A.D. Rollett, The correlation between grain boundary character and intergranular corrosion susceptibility of 2124 aluminum alloy. Appl. Texture Anal. Ceram. Trans. 201, 261-267 (2008)

26. J.G. Brons, G.B. Thompson, A comparison of grain boundary evolution during grain growth in FCC metals. Acta Mater. 61(11), 3936-3944 (2013) 